\title{
St. Petersburg, Andrei Biely
}

\author{
Mercedes JimÉnEZ DE LA FUENTE
}

\author{
I.E.S. Manuel de Falla (Móstoles) \\ merfuente@gmail.com
}

Recibido: 6 de abril de 2011

Aceptado: 13 de mayo de 2011

\section{Resumen}

El propósito de este trabajo es analizar cómo en la obra maestra del escritor ruso Andrei Biely, St. Petersburgo (o Petersburgo), la imagen de la ciudad y de los ciudadanos responde a la configuración temática y formal de la novela modernista. La novela destaca tanto por la belleza de un lenguaje muy poético como por las innovaciones propias de la novela moderna. Se anticipa a otras obras clave dentro de la narrativa del siglo XX. En primer lugar, incluimos una introducción sobre el autor y el movimiento simbolista ruso. Después nos centramos en el texto, analizando el papel relevante de la ciudad como un personaje más, inmortal y activo, y siguiendo con sus habitantes, en una Rusia anterior a la revolución, qué les pasa, cómo viven la experiencia de la vida urbana y cómo están condicionados por el entorno y las circunstancias históricas. La ciudad de Petersburgo aparece cargada de significado.

Palabras clave: novela, Petersburgo, Andrei Biely, Simbolismo, espacio urbano.

Title: St. Petersburg, Andrei Biely

\section{Abstract}

The aim of this paper is to analyse how the image of the city and its citizens in Andrei Biely's masterpiece book, St. Petersburg (or Petersburg), shares with other modernist novels a similar thematic and structural configuration. The novel stands out for the beauty of its poetic language as well as for all the innovations usually ascribed to the modern novel. The work is published earlier than other important works in twentieth-century narrative. This paper begins with an introduction to author and to the Russian Symbolist movement. Later it focuses on the analysis of relevant role of city in St. Petersburg, as another character, immortal and active, and the study of urban figures, in a pre-revolutionary Russia: what happens to them, how they live city life experience and how they are conditioned by their environment and historic circumstances. The city of Petersburg is full of meaning.

Keywords: novel, Petersburg, Andrei Biely, Symbolism, urban space.

\section{Índice}

1. Introducción

2. La construcción del espacio en la novela: la ciudad modernista, un espacio interior

2.1. La ciudad

2.2. Los personajes

3. Conclusión 


\section{Introducción}

St. Petersburg $(1916 ; 1922)$-una de las novelas más importantes del siglo XX, según Nabokov- es considerada la obra maestra de un interesante escritor moscovita que, además de novelista, destacó en la Rusia de principios de siglo como poeta, ensayista, teórico del arte, crítico literario y filósofo, dentro de la llamada "segunda generación" del movimiento simbolista ruso. Boris Nikolaevisch Bugaev (Moscú, 1880-1934) adoptó el pseudónimo de Andrei Biely (también aparece escrito como Bely) a partir de su primera publicación, Segunda Sinfonía, en 1902. La elección del falso apellido Blanco (Biely) puede relacionarse tanto con el especial significado que los simbolistas dan al color, como con el propósito de no comprometer a su padre, Nikolai Bugaev, eminente matemático de la época y decano de la Facultad de Ciencias de Moscú. En 1899 Boris se licenció en Física y Matemáticas por la Universidad de Moscú. Su inquietud intelectual abarcaba muchas disciplinas: la filología, la filosofía, la música, la religión, el misticismo, etc. Su amigo el poeta Aleksandr Blok lo llamaba poeta "genial, extraño". Fue un explorador desasosegado, experimentando eternamente cómo renovar el arte de la palabra, más interesado en el proceso creativo que en el resultado (Maliavina 1997: 1195).

El Simbolismo fue la primera de las corrientes modernistas surgidas en Rusia. Svetlana Maliavina explica que se trató más de un movimiento estético que de una escuela literaria, de hecho su filosofía y su estética estaban en permanente debate dentro del movimiento. Así, los primeros círculos literarios simbolistas se forman en la última década del siglo XIX simultáneamente en dos ciudades: Moscú (donde se crea la editorial Escorpio y más tarde la revista Libra) y San Petersburgo; aunque sus ideas estéticas son distintas, se acaban uniendo posteriormente en el llamado simbolismo mayor. La segunda generación de simbolistas que se formaliza en Moscú (también existe otro grupo en San Petersburgo) con el nombre de los argonautas -a la que pertenece Biely junto a Aleksandr Blok y Serguei Soloviov-, será denominada por la crítica literaria los simbolistas menores. Estos editan la revista El vellocino de oro (1906-1909) para la difusión de sus obras. A diferencia de los mayores, más esteticistas e individualistas, los menores consideran el movimiento como una visión del mundo. Además de la filosofía idealista, su fuente principal es la filosofía apocalíptica de Vladimir Soloviov (la unión positiva, la idea de la síntesis, teurgia). El grupo de los argonautas surgió en un ambiente familiar, entre amigos y parientes que se reunían en casa del sobrino del filósofo, Serguei, bebían té y leían poemas. En 1905 el grupo se traslada a otra casa de San Petersburgo que, por estar situada en el último piso de una torre en la calle Tavrischskaya, será llamada "la torre". Durante un año se celebran estas reuniones todos los miércoles gracias a la generosa 
hospitalidad de los anfitriones: el poeta V. Ivánov y su mujer Lidia. El buen ambiente de este círculo representa, en palabras de Maliavina, "el intento de crear el arquetipo de la existencia humana en las relaciones fraternales, en la franqueza mutua, en el constante y enriquecedor intercambio intelectual" (2002: 133). El cumplimiento del ideal de Soloviov del Simbolismo como la "unión universal" no duró mucho, sin embargo, pues los poetas acabaron polemizando entre ellos.

El Simbolismo poético nace "de la necesidad de adaptar el lenguaje lírico a las nuevas sensaciones del hombre moderno, con la creciente necesidad de reconstruir su relación íntima con lo sagrado y lo trascendente" (Presa González 1997: 1195). Merezhkovski declara en su manifiesto que la nueva poesía precisa del símbolo, elemento capaz, por sí mismo, de profundizar la impresión mística. Para los mayores, el símbolo es una manera de restaurar su relación con lo trascendente. En cambio, para los simbolistas menores es el instrumento que facilita expresar "el movimiento del alma del poeta" y revelar de forma intuitiva el misterio del mundo. Esta época es conocida como el Siglo de Plata de la literatura rusa.

Las primeras creaciones literarias de Biely están determinadas por la filosofía de Soloviov. Andrei Biely, como teúrgo, estaba convencido de que el proceso de creación tenía una misión religiosa cuya meta era la reconstrucción de la vida espiritual de la sociedad. Su primer libro poético, Gold of Azur, se publica en 1904. En esta época escribe sus cuatro Symphonies (publicadas entre 1902 y 1908). Con las sinfonías, género original suyo, Biely intenta crear una nueva forma literaria que combine prosa, poesía y música (a la que considera el arte de las artes), con la que prepara el camino para la prosa rítmica de sus mejores creaciones en prosa (sus primeras novelas). Cuando se distancia del grupo de los argonautas, crece su amistad con Blok, de cuya mujer, Liubov Mendeleev, se enamora. Los filósofos Nietzsche y Soloviov son reemplazados ahora por Kant y los neokantianos. En el libro de poemas Urn (1909) aparecen otros temas más intimistas, como el amor no correspondido, y nuevas formas. Escribe crítica literaria para la revista Libra y, cuando esta cierra, publica tres libros de crítica en la editorial Musaguet: Symbolism, Green Meadow y Arabesques (1910-1911). El tema de la revolución de 1905 aparece en sus primeras novelas, The Silver Dove (1910) y Petersburg (publicada por primera vez en 1913), las cuales iban a formar parte de una trilogía titulada East and West, que quedó incompleta. En esos años el escritor entra en un período de "renovación espiritual" en busca del "camino de la vida". En 1910 se casa con la pintora Anna Turgenieva (sobrina-nieta del escritor Ivan Turgenev) y viajan durante un año por el Mediterráneo. Cuando está trabajando en Petersburg conoce a Rudolph Steiner (1912) y se inicia en la antroposofía, en la que encuentra la encarnación de sus ideales: 
la armonía entre los conocimientos místicos y científicos. En 1913 viaja a Suiza con su mujer y se unen a la Sociedad Antroposófica. Anna no regresará con él a Rusia en 1916. En Suiza había empezado a escribir una novela autobiográfica sobre su primera infancia, incluida la etapa uterina, Kotik Letaev (publicada como libro en 1922). Biely ve en la revolución el camino verdadero de Rusia, el que le corresponde a su predestinación espiritual. Celebra la caída del régimen zarista con el poema Christ Has Risen (1918). Entre 1917 y 1921 trabaja como lector en Moscú y Petrogrado. En 1921 publica su poema autobiográfico The First Encouter. Ese mismo año se separa de su mujer y, tras la muerte de Blok, se traslada a Berlín. Regresa a la Unión Soviética en 1923. Escribe una serie de novelas con el título general de Moscú, de las cuales solo se publican algunas. Muestra interés por el marxismo, sin abandonar sus ideas antroposóficas y a sus amigos. En 1931 se casa con Klavdiia Nikolaevna Vasilea. Escribe una trilogía de memorias sobre la vida cultural y social de Rusia antes, durante y después de la revolución (1930-1934). Su última obra es The Mastery of Gogol (1934), un detallado estudio del lenguaje y el estilo de Gogol. A su muerte, gran parte de sus escritos quedaron sin publicar.

St. Petersburg fue revisado y reelaborado en varias ocasiones por el autor. Biely escribió el primer texto entre junio de 1911 y enero de 1912. Rechazada por los editores, siguió trabajando en la novela hasta que en 1913-1914 fue publicada por entregas en el almanaque Sirin, y más tarde en forma de libro, en 1916. No satisfecho totalmente con el resultado, siguió revisándolo. La nueva versión se publicó en Berlín en 1922. La novela no se editó en Rusia hasta 1928.

La primera edición en inglés data de 1959; la novela rusa fue traducida por John Courton con el título de St. Petersburg o Saint Petersburg. En la introducción solo se menciona la versión de 1913, lo que nos hace suponer que es en la que está basada la traducción del escritor inglés, y esta es la que hemos manejado en este trabajo. Otras adaptaciones al inglés son la traducción de Malmstad \& Maguirre (Indian University Press, 1978), muy prolija en anotaciones; la de David McDuff en Penguin (1955), que traslada la versión de 1922, y la traducción de John Elsworth (Pushkin Press, 2008), que vuelve a la edición de 1916. 


\section{La construcción del espacio en la novela: la ciudad modernista, un espacio interior}

A consequence of a pluri-dimensional discontinuous perception of the world, the study of time-simultaneity gave way to theories as distinct as Einstein's relativity or Bergson's subjective time. It becomes the object of modernist experimentation in the works of literary figures such as Marcel Proust, Virginia Woolf, James Joyce or Thomas Mann. (López-Varela 2010: 5)

El especial énfasis en la representación del espacio y del tiempo que ponen los modernistas "manifiesta el deseo de capturar el fenómeno temporal en un marco espacial" (López-Varela 2009: 5). Si los escritores modernistas sitúan en primer plano la construcción del espacio y del tiempo como coordenadas que enmarcan las acciones de los personajes, estas vienen a representar los movimientos del ciudadano, sobre todo mentales, relacionados con los nuevos tiempos y la problemática del individuo moderno. Petersburgo es una novela de principios del siglo $\mathrm{XX}$ que podría compararse con otras novelas modernistas que también experimentan con el tratamiento del tiempo y del espacio; de hecho, se ha relacionado sobre todo con Ulysses de James Joyce y con Berlin Alexanderplatz de Alfred Döblin: la acción se desarrolla durante uno o varios días en una única ciudad recorrida por los personajes, la cual tiene tanto protagonismo como ellos; todo lo que ocurre está encuadrado dentro de un marco urbano que determina en cierta manera los sucesos, porque son esa ciudad y sus circunstancias, y no otras posibles, las que condicionan el vivir de los habitantes; la presencia del humor es importante. Biely comprime las peripecias de unos personajes durante el último día de septiembre y en unos pocos de los primeros días de octubre en el San Petersburgo de 1905. Alexander y Nikolai, protagonistas de la novela, se sorprenden cada uno de todo lo que les ha ocurrido en solo veinticuatro horas. No se trata tanto de acontecimientos externos como de fuertes y poderosas emociones que los llevan a comportamientos extremos, dudas exageradas o estados de delirio. Ellos reaccionan ante lo que experimentan vitalmente, y son justamente los pensamientos y las acciones consecuentes el objeto de la narración. Sin embargo, a diferencia del Ulysses de Joyce o de Mrs. Dalloway de Virginia Woolf, por ejemplo, cuyo tiempo interno es un día y el paso de las horas va siendo minuciosamente señalado por el narrador (un reloj suena, etc.), en Petersburg no solo no encontramos apenas referencias temporales durante los pocos días, reducibles a horas, en que transcurre la acción, sino que hay una deliberada intención de buscar la imprecisión en el tiempo. De esta forma, las escenas adquieren un carácter borroso, casi onírico. Si los personajes son sombras, seres que surgen de la nada para adoptar una forma humana, sus acciones son retazos de sueños o de 
pesadillas, fragmentos de tiempo. Los únicos datos explícitos aparecen al principio de la historia: ésta comienza el último día de septiembre a las ocho y media de la mañana, hora en que el Senador Apollon Ableukhov se prepara para ir al Negociado; camino del trabajo, en un coche negro por las calles de la ciudad, un cañonazo marca las doce. No vuelve a hacerse ninguna mención concreta a las horas ni a fechas precisas. La naturaleza será quien nos indique, con sus bellos amaneceres y sus atardeceres, el paso del tiempo. Los acontecimientos del primer día son contados en el primer capítulo y en parte del segundo; los sucesos de algunos días después (no hay datos que permitan precisarlo, se habla de "frosty days of early October") ocupan los capítulos dos y tres; en el capítulo cuarto y en parte del quinto se narran los hechos de un día de octubre; los capítulos siguientes, del quinto hasta el octavo, recogen la acción del día después, que es el último. La dilatación del tiempo a lo largo de, prácticamente, toda la segunda mitad de la novela se corresponde con un momento de máxima intriga: Nikolai Ableukhov ha activado una bomba que explotará en un plazo de veinticuatro horas. Los acontecimientos se precipitan.

Petersburg también comparte con otras novelas de principios de siglo la ruptura con la narración tradicional en lo que se refiere al orden del discurso. La historia no se cuenta de forma lineal y como un todo, sino que se presenta fragmentada, dividida en partes que se yuxtaponen entre sí, en ocasiones como secuencias simultáneas o desordenadas en el tiempo, sugiriendo caos. George Reavey escribe en el prefacio de la edición de Courton: "It is an extraordinary novel conceived on many levels and deliberately constructed to produce an impression of chaos and nightmare" (Biely 1987: vii). López-Varela explica cómo esta técnica, relacionada con el novedoso tratamiento del tiempo y del espacio, obliga al lector a componer la historia contada: "in Ulysses he [Joyce] defines the city as aggregate of parts where the co-present of the city inhabitants and their simultaneous errands offer the portrait of the modern fragmented metropolis where humans are only able to apprehend a small portion of this urban space that the narrative's modernist techniques render whole only to the reader who can trace the spatiotemporal relations to other urban figures" (López-Varela 2010: 7). Pero a diferencia de Ulysses o Mrs. Dalloway, por nombrar los ejemplos del artículo citado, en la novela de Biely el empleo del monólogo interior y de la corriente de conciencia no se adueña totalmente del discurso, sino que aparece en determinadas secuencias focalizadas en los personajes sobresalientes; en realidad, el narrador de Petersburg está muy presente en la narración. Se trata de un narrador omnisciente muy conversador y subjetivo. Atendiendo a las funciones del narrador establecidas por G. Genette (1989), hace uso tanto de la "función de control" como de la "función de comunicación": la primera aparece 
cada vez que el narrador alude a la articulación de la organización interna del texto; con la segunda establece un diálogo con el lector, auténtico "narratario" del discurso. Al final del primer capítulo interviene filosofando sobre la creación; dice que si bien un ser de ficción es producto de la fantasía del autor, un "mere idle cerebral play", una vez creado comienza su existencia propia porque "thoughts too have their own particular existente" (Biely 1987: 38). Esta idea del pensamiento como juego cerebral se repite a lo largo de su novela. Veremos más abajo cómo los personajes son sombras que van tomando consistencia humana, cómo se concede una gran importancia a la descripción de las sensaciones y los delirios mentales de algunos de ellos, al límite entre la percepción subjetiva y otra realidad que va más allá de la realidad física. La voz autorial está también muy presente, llegando a expresar experiencias autobiográficas del propio escritor.

Tres historias interrelacionadas entre sí componen Petersburgo:

1. Historia de los Ableukhov: Nikolai, Apollon y Anna.

2. Historia amorosa de Nikolia y Sofya.

3. Historia del dirigente revolucionario que vive en la clandestinidad, Alexander, relacionado con Lippanchenko, verdadera encarnación del mal.

La primera es el relato de una familia desintegrada que, cuando se le presenta la posibilidad de volver a unirse, tiene en contra al azar y se separa definitivamente bajo los efectos de una bomba que inoportunamente estalla al final de un día presidido por la angustia de quien la ha activado sin intención real de hacerlo. El hijo dejará el hogar familiar y el país para no volver hasta la muerte de sus progenitores. Se siente culpable por haber desencadenado el complot del atentado contra el padre al ofrecerse a consumar él mismo la acción, meses atrás, ante un grupo de revolucionarios. La segunda cuenta el desengaño amoroso de Nikolai, enamorado de una mujer casada, Sofya Petrovna. El romance empezaría el mismo día de su boda con el teniente Serguey Likhutin, amigo íntimo de Nikolai. El joven se siente muy ofendido cuando ella, una dama superficial y de limitada inteligencia, lo rechaza. Decide tomar venganza y burlarse de ella, y ella, a su vez, se vengará de él. La tercera se centra en uno de los personajes más interesantes de la novela, el ex preso político Alexander Ivanovich Doodkin. Por consejo de Lippanchenko, un malvado activista de confuso origen, vive escondido en una paupérrima buhardilla situada en la Línea Decimoséptima de la Isla Vasilyevsky. Este hombre de notable inteligencia y cultura va siendo destruido poco a poco a causa de la miseria en la que vive, presa del insomnio y de las alucinaciones, arrastrado al alcoholismo por su falso protector, de quien no es más que una víctima. Desde el principio sabe que el destino de otros pensadores comprometidos como él no es otro que la locura. 
Igualmente pude ser útil un breve resumen del argumento.

La pésima relación padre-hijo es uno de los temas principales de la novela. Nikolai, un aventajado estudiante de Filosofía, hijo del odiado Senador Apollon Ableukhov, se ve involucrado en un intento de atentado contra su padre. Son prácticamente dos extraños que viven juntos desde que su madre, Anna, los abandonó hace dos años. Incluso sienten una gran hostilidad mutua. A principios de otoño el joven, trastornado por un desengaño amoroso, ha olvidado la promesa que hizo al Partido en verano de ser él mismo quien ejecutara el asesinato. La agitación social está en la calle, se reparten panfletos contra el poder, se celebran mítines, se reúnen los conspiradores contra el poder del zar; todo anuncia un nuevo brote de la revolución de 1905 . La trama de la novela comienza, pues, in medias res. El primer día en el tiempo interno, treinta de septiembre, narra el primero de una serie encadenada de acontecimientos. Lippanchenko cita a Alexander en un restaurante para entregarle un hatillo con la bomba que ha de llevar a Nikolai, junto con una carta donde se le recuerda la palabra dada. Camino del restaurante, Alexander se ha cruzado con Apollon y lo ha reconocido; también el anciano se ha quedado impresionado por los ojos del único transeúnte que se atreve a mirarlo dentro del elegante coche de caballos con el que se dirige al Negociado. Mientras tanto, los pensamientos del joven aristócrata están ocupados en cómo vengarse de la dama que lo ha rechazado, días antes, llamándolo "payaso rojo". Se trata de Sofya Likhutina, también relacionada con los revolucionarios. Alexander llega a casa de Nikolai y le entrega el envío (una lata de sardinas que contiene el explosivo), pero olvida la nota. Los días siguientes es noticia un desconocido vestido de dominó rojo que se aparece en los lugares más inesperados: es la respuesta de Nikolai ante el agravio sufrido. Con este disfraz asusta a Sofya, quien reacciona planeando entregarle la carta, llegada a sus manos a través de un miembro del Partido, y, vestida de la Pompadour, asiste al baile celebrado en casa de los Tsukaton. En la fiesta aparecen el dominó rojo y el jefe del Negociado. Nikolai, al leer la nota, huye precipitadamente y deambula pensativo y asustado por la ciudad durante toda la noche. Apollon también pasea por las calles antes de regresar a su casa, tras enterarse de la identidad del dominó rojo y de ser informado de lo que se planea contra él. Padre e hijo llegan casi a la vez a su hogar. La inevitable conversación entre ellos no se produce por un imprevisto: la noticia de que la madre ha regresado. El joven Ablekhov, excitado por la misión encomendada e incapaz de hacer nada, va en busca de Doodkin, quien empatiza con él e intenta ayudarlo enfrentándose a quien resulta ser el verdadero responsable del plan, Lippanchenko. Pero una vez más es vencido por este horrible hombre, que se apodera de su voluntad. Al final del día, en pleno delirio, Alexander regresa para asesinarlo. Durante ese tiempo 
Nikolai continúa perdido por la metrópolis hasta que Serguei Likhutin, reconciliado con su esposa, se lo lleva a su casa para evitar que cometa el atentado. Cuando por fin Nikolai consigue llegar a su mansión, ya ha tomado la decisión de destruir el artefacto, mas es demasiado tarde: en su ausencia su padre se lo ha cogido sin saber qué era. El tiempo corre en su contra pues había activado la bomba antes de salir. De madrugada, después de una velada familiar conciliadora gracias a la presencia de la madre, se oye una explosión en el estudio del senador. Solo produce daños materiales; sin embargo, el futuro común de los Ableukhov ha terminado.

\subsection{La ciudad}

En St. Petersburg el espacio está siempre presente en la narración. Son contadas las escenas donde no se hace alguna referencia al entorno, a los monumentos relevantes, al momento del día o a la condición atmosférica, si llueve, hay bruma o niebla, empiezan las primeras nieves o hay humedad en la ciudad.

En los espacios exteriores no se detalla cómo son los elementos constituyentes de la ciudad (los espectaculares monumentos, las calles y avenidas, los puentes, los ríos, los jardines), sino que la atención va dirigida al momento del día que abre o cierra la escena, al tiempo atmosférico, a cómo son las calles. Las poéticas descripciones de los amaneceres y de las puestas de sol van marcando el transcurso del tiempo, en ausencia de marcas temporales. Algunas alusiones al entorno no son más que ligeros apuntes: "After the slush melted, the Petersburg roofs were bathed in sunlight" (Biely 1987: 82); "The last leaves, golden and red, rustled in a neighboring garden" (Biely 1987: 85). Otras veces se trata de breves pinceladas al inicio o al final de los subcapítulos, hermosos cuadros creados con palabras:

Tuft of clouds drifted in a greenish swarm, moving without pause over the boundless expanse of the Neva's broad embankment. From the Petersburg side, the slender spire of Peter and Paul vanished into the clouds.

A dark strip of soot, rising from the chimneys, described a funereal arc and dipped its tail in the water.

The Neva seethed; the siren of a small passing steamer sounded clamorously, the steel sides of ships beat against the piers and lapped the granite of the embankment. (Biely 1987: 31)

La plasticidad de las descripciones se consigue con la atención al color, así como la selección de un léxico suntuoso: "dim turquoise shadows spreads soundless under foot [...] the turquoise air became imbued with gloom" (Biely 1987: 191); en la avenida Nevsky, "along the sides placed the constantly changing gleam of shop signs; here, 
rubies off lame suddenly flashed and vanished; there, emeralds as suddenly sparkled and disappeared" (Biely 1987: 33). La ciudad parece ir surgiendo al paso de los recorridos de los personajes, como en la escena inicial que sigue a Apollon dentro de su coche de caballos camino del Negociado, o la escena del capítulo tres que acompaña el camino de regreso de las dos amigas, Sofya y Varvara:

Hanging, as it were, above the dense mist, there first appeared the indeterminate shape of St. Isaac's Cathedral, as the carriage sped onward, gradually descended earthward; then out of the mist emerged the equestrian statue of the Emperor Nicholas and, at its foot, a grenadier of Nicholas's day with a shaggy bearskin on his head. The carriage now sped along the Nevsky. (Biely 1987: 10)

They had reached the Embankment: the deep water of the Neva looked green-blue. Far, far away, appearing ever so remote, the islands looked incredibly flat and low; and their buildings seemed so flat too that the deep green-blue waters threatened suddenly to wash over and submerge them. A pitiless sunset hovered above this greenblue surface, scattering its gleams here and there: the Troitsky Bridge and the Winter Palace glowed purple. (Biely 1987: 86)

Los elementos más importantes del Petersburgo de Biely: la catedral de San Isaac, el puente Moika, el puente Troitsky, el Palacio de Invierno, la estatua ecuestre del emperador Nicolás, el Jinete de Bronce (Peter I, fundador de la ciudad), la avenida Nevsky, el río Neva, la aguja de Pedro y Pablo, el Jardín de Verano son citados continuamente como si se tratara de entidades que están presentes en los acontecimientos, de forma que la ciudad parece estar viva y ser testigo de cómo se las ingenian sus habitantes; es mucho más que el escenario de sus acciones, crea la impresión de un organismo vivo que recoge en su seno a individuos muy pequeños frente a su inmensidad, además de vulnerables y perecederos. La idea de la muerte está presente desde las primeras líneas de la narración: en la primera salida de Alexander a la calle, las hojas que observa "immersed in these thoughts, the stranger clenched a fist in his pocket; and then he suddenly though of the falling leaves. He knew it all by heart. For many, these falling leaves were the last leaves" (Biely 1987: 14); más tarde, las hojas arremolinadas le hacen pensar en que vendrá una época sangrienta; Nikolai recuerda cómo ha cruzado por su mente la idea del suicidio al pasar por uno de los puentes de la ciudad; en varias ocasiones su imaginación anticipa de una manera incluso morbosa cómo el cuerpo de su padre saltará en pedazos (se encontrará una tibia por aquí, sesos por allá) ante el efecto de la explosión.

El paisaje cobra vida a través de constantes personificaciones: "The palace flamed blood-red" (Biely 1987: 115); "The Neva seethed 
and foamed under the pressure of the moist wind" (Biely 1987: 36); "High up, arms shaped from clouds ascended above the tumultuous waters of the Neva, racing toward the zenith; and, when they touched the zenith, the phosphorescent stain fell upon them" (Biely 1987: 36). Por el contrario, las personas se animalizan o se cosifican: la multitud es "a polypood"; respecto a Nikolai, "his tragic mask contradicted his lizard-like shiftiness" (Biely 1987: 197); de Lippanchenko; "Despite his resemblance to a rhinoceros" (Biely 1987: 276).

Los protagonistas aparecen solitarios en el marco de la ciudad, como seres que deambulan por sus calles, contemplan el río o la luna, en comunión con la ciudad. Las acciones los sorprenden caminando o desplazándose. Se encuentran unos con otros fortuitamente. Se citan en lugares públicos. Se esperan. Se despiden.

San Petersburgo toma su verdadera forma bajo los pasos de los caminantes que la recorren con admiración o desagrado. Es hermosa para los aristócratas, habitantes de la bella ciudad imperial; no lo es tanto para quienes viven en Vasilyevsky Island, trabajadores, revolucionarios o marginados. Según De Certeau, empezamos a hablar de la ciudad con los pasos, un número que no forma serie ni se puede contar: "Su hormigueo es un innumerable conjunto de singularidades. Las variedades de pasos son hechuras de espacios. Tejen los lugares [...]. No se localizan: espacializan" (De Certeau 1994: 5).

Los trayectos de nuestros personajes simbolizarían las venas que dan vida al organismo que es la metrópolis, más allá de un simple espacio físico -lugares que podríamos localizar en un mapa-, es la manera en que los petersburguenses viven en ella y cómo la viven en relación a sus problemas y realidades. Los personajes más importantes tienen sus propios recorridos:

1. Los paseos nocturnos de Nikolai, vestido con el dominó rojo, esperando a Sofya o contemplando el puente desde donde una vez pensó en suicidarse; delirante cuando lee la nota en la fiesta o cuando va en busca de Alexander para culparle de haberle llevado la bomba. El último día parece ir de un sitio a otro, de la casa de Alexander a la universidad, deshace lo andado, se queda parado frente a las vitrinas de las tiendas, llevado por el estado de angustia que le provoca la cuenta atrás de los explosivos. Es una mezcla de flâneur y de exhibicionista.

2. A Sofya le gusta deambular por las calles. Cuando espera ansiosa la llegada de Nikolai, después de su desencuentro, limpia el cristal de la ventana con un delicado pañuelo y lo busca. Al no verlo, sale: "Deceived in her presentiment, Peri [Sofya] bit the damp handkerchief, and ran to put on her fur coat and hat; pressing her 
little nose into her muff, she sauntered from Moika to the Embankment" (Biely 1987: 50). Le calma andar después de salir nerviosa de una reunión de espiritismo celebrada unos días antes. También le agrada contemplar la ciudad de noche desde su ventana o salir a la calle para observarla desde un puente próximo a su casa.

3. Alexander necesita la calle para aclarar su mente después de los delirios nocturnos o para dejar por un tiempo la pensión miserable donde vive; también cuando se siente sobrecargado por el humo o el alcohol. Así, tras la primera larga e intensa conversación que mantiene con Nikolai en su mansión (capítulo segundo), le propone salir a airearse para descongestionar sus pulmones y su cabeza. En el capítulo seis, focalizado en este personaje, se comenta cómo tras una mala noche siente la necesidad imperiosa de dejar su buhardilla y lanzarse a la calle:

Alexander I. D. surveyed his habitation; he longed to be outdoors, in the unclean fog, rubbing shoulders, mingling with other backs and greenish faces, on a Petersburg prospect. [...] He felt a desire to be overwhelmed by the fog, to drown in it the nonsense which irritated his brain, to extinguish the surges of delirium by exercising his legs. He wanted to stride from prospect to prospect, from street to street, until his brain was numbed, and the collapse at a tavern table and burn his organs with vodka. (Biely 1987: 190)

En este capítulo ocurre la segunda conversación entre los dos jóvenes, tan larga como la primera. Su diálogo se desarrolla al compás de sus pasos por la ciudad. Nikolai sorprende a su cómplice cuando este está bajando las escaleras de su casa en busca de la calle, y se une a su paso sin rumbo fijo. Alexander conoce tan bien la rúa que se da cuenta de sus más mínimos cambios; esta vez piensa que todo presagia la revolución (de hecho es el día previo a la huelga). Agobiado por el aristócrata parricida, llega un momento en que desea continuar solo su camino para poder reflexionar.

4. El senador recorre las arterias de la ciudad en su coche negro tirado por caballos. Solo en una ocasión dejará el vehículo, tras la noche de la fiesta de disfraces, cuando conmocionado por lo ocurrido decide andar por la ciudad. En este paseo entra por primera vez en los barrios pobres que suele evitar y por los que siente una profunda aversión; así, el caminar a pie, bajarse del lugar que le sitúa por encima de los demás aislándolo, se convierte en un símbolo de su acercamiento a los humanos menos favorecidos socialmente. Las terribles noticias le hacen más humano por un tiempo. Una vez en su casa, volverá a convertirse en el padre autoritario que desconfía de su hijo, hasta que la huida definitiva de este reblandezca 
su corazón y le haga un padre siempre anhelante y deseoso de que lleguen noticias de su vástago, al que nunca volverá a ver.

También interesa la visión de algún personaje que destaca de la masa:

5. Un obrero, Stepka, recorre a pie el camino desde Kolpino, donde está la fábrica de la que ha sido despedido, hasta San Petersburgo. El paisaje descrito no puede ser más desolador: "the very earth looks like a corpse", "a place of many chimney and much grime" (Biely 1987: 73). Este trayecto muestra la fealdad de la ciudad industrial.

San Petersburgo es una ciudad de los nuevos tiempos: por un lado, sigue sosteniendo la sede del poder zarista, representado por el Negociado, lugar al que acude diariamente en coche tirado por caballos el burócrata Apollon Ableukhov, senador aspirante a ministro; pero, por otro, las avenidas se iluminan con luz eléctrica, los automóviles cruzan veloces y allí se levantan a modo de cinturón las industrias humeantes, así como los barrios obreros situados en las islas, a una oportuna distancia del centro histórico. Estamos en la Rusia de la revolución de 1905, los campesinos que se levantan contra los señores e incluso queman sus viviendas, los obreros que reclaman mejores condiciones de trabajo al zar, los intelectuales que se unen a la petición de una sociedad más justa. Sin embargo, el contexto histórico se dibuja como telón de fondo, desprovisto de contenido político, mostrado brevemente con suaves y poéticas pinceladas:

The ploughmen had already ceased scraping the soil [...]; the long country nights flared with incendiary fires.

That was the situation in the villages.

It was the same in the towns. In the workshops, in the printing shops, in the hairdressing establishments, in the dairies, in the taverns, everywhere there was the same ubiquitous garrulous person; fresh from the blood-soaked fields of Manchuria, a shaggy cap pulled over his eyes, a Browning in the pocket, the thrust badly composed leaflets into people's hands.

Everyone seemed to be afraid of something, everyone seemed to hope for something; people poured into the streets and formed crowds, and then scattered again. (Biely 1987: 576-579)

St. Petersburg recoge el ambiente previo a la huelga de principios de octubre. Las manifestaciones contra el poder zarista que bullen en todo el país llegan a sus contornos, tocando no solo a los trabajadores (Stepka) sino también a los jóvenes de las clases privilegiadas, intelectuales (Nikolai) y damas de sociedad (Sofya). La agitación es descrita como un ente vivo que va penetrando en todos los lugares de la urbe. Los acontecimientos históricos transcurren 
paralelos a los personales, de manera que el mismo día que la bomba está activada y explotará, estalla la huelga y Apollon deja su cargo. En una misma jornada se destroza su carrera política y su vida familiar, pues pierde definitivamente sus ambiciones políticas y a su hijo.

San Petersburgo es asimismo la ciudad imperial, capital de Rusia en 1905, construida a imitación de las capitales occidentales por el zar Pedro el Grande en 1703. Sus líneas rectilíneas, las grandes avenidas, los cruces siguen la planificación de las ciudades europeas. Nuestra novela comienza con un prólogo que presenta a San Petersburgo, con la ironía característica del narrador, como una ciudad más del inmenso Imperio Ruso, pero que difiere de todas las demás por su trazo organizado, del que es ejemplo la rectilínea avenida Nevsky. De esta forma se establece una oposición entre occidente y oriente, así como entre San Petersburgo y Moscú, con mayor número de habitantes.

Existe otro Petersburgo soñado o irreal en el que parecen cobrar vida las estatuas: el Jinete de Bronce, Pedro I, desaparece de su pedestal posiblemente para pasearse por la ciudad creada por él, 0 el Atlante o "barbudo de piedra" del Negociado sonríe ante los avatares de la existencia humana. Una urbe misteriosa donde los habitantes son protegidos por un ser sobrenatural que bien podría corresponderse con el Holandés Errante. Un espacio que se abre a otros espacios insondables o "inabarcables", mentales, que conocen Apollon, Nikolai o Alexander, y de los que hablaremos más abajo (en lo que se refiere a Apollon, véase la última parte del capítulo tercero, por ejemplo).

Por último, también está el San Petersburgo del autor, aquel al que evoca la voz autorial recordando cómo en el mismo puente donde Nikolai se para estuvo también él pensando en suicidarse (capítulo cinco, ediciones posteriores). El mismo que pregunta al lector si fue testigo de la revolución como él, del ruido que se oía por todas las ciudades rusas en los días previos a la huelga: "a persistent moan with stress on the note oo"; "Did you happen to hear this October song of the year 1905?" (Biely 1987: 58).

\subsection{Los personajes}

Con la excepción de Apollon y Nikolai, los personajes aparecen por primera vez como seres comunes, todavía sin nombre propio, en los que "se fija" el narrador y de los que destaca alguno de sus rasgos característicos. Esta forma de introducir un nuevo personaje resalta su condición de seres cualesquiera (uno más de la masa humana) surgidos repentinamente. En realidad, los personajes son sombras que emergen de la bruma o se ocultan en ella cuando caminan por la ciudad. Como explica el narrador, es algo inherente a la propia ciudad: "The streets of Petersburg posses one indubitable quality: 
they transform the figures of passes-by into shadows" (Biely 1987: 23) y las sombras en personas. Así, Alexander es "a strange" que se convierte en sombra:

The stranger became a bluish shadow. Shadows swarmed across the bridge; the stranger's dark shadow was one of them. From this shadow's hand a bundle dangled rhythmically, a bundle neither small nor large. (Biely 1987: 14)

En las primeras páginas de la novela Alexander es nombrado como "the stranger with the tiny black mustache". Lippanchenko y otro hombre que lo acompaña aparecen por primera vez como "two silhouettes", poco después como "two shadows" y el primero será "the person" (la cursiva es del autor) en la escena final que revela toda su negatividad. Sofya, antes de ser presentada, es "a woman's shadow" que se desplaza a lo largo del Moika. Stepka es "a worker". También la madre fugitiva que regresa aparece ante la casa como "a medium-sized plump woman, all in black". Las referencias a Nikolai son varias: el "red clown", "a figure in an Italian cloak and a fantastically bent hat".

Los personajes se van materializando a través de una progresiva descripción que comienza por su rasgo más característico, las ropas que visten o su constitución física. Se asemejaría a un proceso de creación: de la nada al detalle y del detalle al todo, con el nombre, como si de un bautizo se tratara, al final. Otro recurso para referirse a los individuos es mediante sinécdoques: en su cruce de caminos de las primeras escenas de la novela, la imagen de Apollon con que se queda Alexander es una calva y una oreja y, a su vez, el primero solo recuerda el bigote negro del segundo; Sofya se fija en alguien que se le aparece en la calle: "a cylindrical hat, a walking stick, a greatcoat, ears, and a nose" (Biely 1987: 93). En los agitados días anteriores a la huelga, cuando la intranquilidad y el miedo de los ciudadanos es evidente, se observa que en la avenida Nevsky "the cylinder hats" han disminuido $y$, en cambio, han aumentado "the black chaggy cap" de la sublevada Manchuria. Justamente la metonimia y la animalización son los recursos con que es descrito un personaje colectivo nombrado arriba: la masa humana. La multitud que pasea por la calle está formada por sombreros o narices: "A great swarm of bowlers and cylinder hats rolled along the streets toward them [...]. Noses were thrust forward everywhere: eagle noses and cock noses; duck noses and hen noses; a green nose and a red nose" (Biely 1987: 198). En el capítulo sexto encontramos la mejor caracterización de este personaje colectivo, observado a través de la mirada de Alexander, quien es arrastrado hacia la avenida Nevsky por una masa representada por la unión de muchos hombros: 
The crowd of shoulders formed a cohesive and slowly moving mass [...]. The bodies of those who were transformed into one general body, like grains of cavier. The pavements of the Nevsky formed a sandwich field where individual thought became part of the general thought of the polypedal creature which crawled along it. (Biely 1987: 198)

Se insiste aquí en la idea de que no hay individuos sino un todo que avanza lentamente: "a multiple feet", "growling polypood", "a variety of voices". Es una masa viva terrible, que permanece a través de los siglos, que no tiene límites y que se extiende a lo largo de toda la avenida: "It was always the same: its head was curled beyond the railway stations; its tail was thrust into the Morskaya" (Biely 1987: 199).

También es importante el empleo de la elipsis, bien como forma de hacer avanzar la acción seleccionando tan solo los hechos que se quieren destacar, bien como un recurso para crear intriga. Una larga conversación entre Alexander y Nikolai ocupa gran parte del capítulo segundo; tras horas de hablar, fumar y beber los amigos deciden salir a la calle con el fin de despejarse; a continuación seguimos el camino emprendido por Alexander sin saber cuándo ni dónde se han separado. En una escena del capítulo tres se omite el mitin al que acuden los jóvenes revolucionarios. El contenido de la carta dirigida a Nikolai no se desvela hasta muy avanzada la narración, de manera que se mantiene el suspense sobre la parte central de la trama, la acción terrorista del hijo contra el padre.

La descripción de los espacios privados de los seres de ficción se hace de forma selectiva, prestando atención a los objetos que mejor definen a estos y al color. En la mansión de los Ableukhov predomina el gris; en el estudio de Nikolai sobresale el verde; en la casa de Sofía, el rojo. Se insiste en que Alexander vive en una inmunda buhardilla, miserable y repelente, lugar de soledad y aislamiento que alimenta sus alucinaciones y pesadillas. Sofya vive en un apartamento de la Moika donde todo señala la falta de perspectiva: los cuadros con paisajes japoneses carecen de ella, las habitaciones las ocupan objetos enormes; en la decoración predominan los detalles orientales (abanicos, crisantemos, cortinas de bambú) e incluso ella parece una japonesita, pero los objetos se acumulan Ilenando las salas. Un detalle relacionado con el espacio, el no tener perspectiva, caracteriza a una dama corta de miras. A Apollon, la figura del poder y del orden establecido, le fascina la geometría y ve el espacio con formas poligonales y líneas rectas; ama la simetría. En su despacho los armarios y los libros estaban colocados en perfecto orden y en la mesa del centro se encontraba un manual de planimetría. 
El narrador no recurre tanto a la omnisciencia para retratar a los personajes como a la mostración de sus acciones y palabras. Las analepsis que explican un suceso del pasado reciente o más lejano o el porqué de un comportamiento del presente aparecen como escenas oníricas que se integran en la narración sin marcas que las abran o las cierren. El participativo narrador no se ahorra adjetivos, símiles o ironías para ponen en evidencia sus defectos, o incluso ridiculizarlos. Cuando es así, más que acercarse a individuos de carne y hueso, los personajes se aproximan a caricaturas: el poderoso envejecido y perdedor (Apollon), el hijo rebelde pero pusilánime (Nikolai), la dama bonita y superficial (Sofya), el marido ultrajado (Likhutin), el malvado sin escrúpulos (Lippanchenko). Por ejemplo, el consejero imperial no solo es ridículo por sus grandes orejas, dibujadas en las octavillas callejeras, su minúscula estatura, la poca gracia de sus bromas, la costumbre de sentarse formando con sus piernas un ángulo de noventa grados, sino porque no se le ve entre los demás funcionarios, tan viejitos como él, en una celebración de estado, o por cómo reacciona el día de la huelga quedándose en casa para limpiar el polvo de los libros. La reducción de estos seres a meros títeres es evidente en la escena central del encuentro del padre y el hijo en su casa tras el baile (capítulo quinto, 6); Apollon ve reflejado en los espejos de su habitación "a marionette", en quien reconoce a Nikolai. Sin embargo, el conflicto generacional adquiere un tono serio e incluso trágico al describir la dureza con que cada uno de ellos juzga al otro. La incomprensión es absoluta; se consideran mutuamente unos canallas. El padre no reconoce en Nikolai al hijo que educó. Este detesta a su padre hasta el punto de desear su muerte, aunque después se arrepienta de la palabra dada, y cuando la furia del progenitor contra él es mayor, lo imagina destrozado por la bomba.

Pero no olvidemos que la ciudad es "more than just a physical structure. It is, among other thing, a state of mind, an order of morality, a pattern of attitudes and ritualized behaviour, a network of human connections, and a body of customs and traditions inscribed in certain practices and discourses... as its etymology suggests, the city is both internal and external" (Zhang 1996: 3-4, 6). Así, en esta novela moderna, no importan tanto las acciones como las sensaciones y las emociones que van experimentando los protagonistas, puesto el foco de interés en su interior. Sus pensamientos y sus emociones se proyectan en el espacio, incluso las alucinaciones sufridas cuando atraviesan un estado crítico debido a la angustia, el alcoholismo o el estrés.

Alexander reflexiona sobre los acontecimientos que le han sucedido en este último día de septiembre mientras camina de regreso a su casa a lo largo del Neva (capítulo segundo, 14). Las detalladas descripciones de los estados anímicos por los que pasan 
los personajes son uno de los mayores logros de la novela; incluso llegan a adquirir forma física:

\begin{abstract}
Alexander Ivanovich's experiences of the day trailed behind him like an invisible tail. A. I. lived his experiences in reverse order, for they retreated behind his back. His back seemed to open like a door and, out of that door, a giant body was poised to pounce: this giant embodied he sum total of his experiences within the past twenty-four hours. (Biely 1987: 71)
\end{abstract}

En el capítulo sexto, clave en la caracterización de este personaje, vemos cómo son plasmadas las sensaciones del despertar y del malestar provocado por una mala noche en alguien debilitado y enfermo. Así: "The night had been an event of gigantic proportions", "The transitional state between dream and waking felt like a jump from a fifth-story window" (Biely 1987: 189). En la escena de la conversación que mantiene con Lippanchenko en su casa, la naturaleza vuelve a hacerse importante. Las constantes referencias al paisaje que se ve desde la ventana parecen ser el único respiro en ese detestable lugar. El contraste entre la belleza de la puesta de sol y la fealdad física y moral de Zoya y su esposo es evidente. Así, "The cottage windows faced the sea. The atmosphere was a deepening blue"; "They could see the foaming waves. A bout rocket in the blue twilight; its sharp-winged sails cut the darkness; the bluish night thickened around them" (Biely 1987: 207-208). Al final, tras ser vencido por el falso hombre, siente que la oscuridad de la noche también ha penetrado en su alma: "Darkness had fallen [...]. Every thing receded into the darkness [...]. Darkness had entered his soul: he was crying" (Biely 1987: 218).

Otro ejemplo de la proyección de la subjetividad en el espacio o en los acontecimientos lo encontramos en la larga secuencia del baile de disfraces. Nikolai huye de la fiesta muy afectado y, en la calle, es perseguido por Morkovin, un ambiguo ser que juega con varias identidades, aumentando su confusión. Una vez más, los monumentos de Petersburgo acompañan el camino de la pareja hasta un restaurante. Nikolai observa que ha desaparecido el Jinete de Bronce. Mientras cenan, el extraño presiona al joven. Cuando sale del restaurante trastornado por el cansancio y la conversación, la ciudad impone su presencia ante él como un personaje más. Ante su mirada, el Jinete reaparece (justifica su ausencia diciendo que antes le cubrían las sombras, si bien el lector lo imagina recorriendo su ciudad) y parece cobrar vida, sonreír e incluso hablar. Nikolai interpreta que es la ciudad quien le recuerda que debe cumplir con una obligación. En el capítulo siguiente vemos cómo activa la bomba casi inconscientemente, $\mathrm{y}$, a continuación, entra en un estado de delirio, obsesionado por la misión que ha de cumplir. El joven parece 
entrar en contacto con otra dimensión, ya intuida cuando imaginaba que al otro lado de las puertas no había nada sino el cosmos infinito. Ese día extrañas apariciones proceden del cosmos, seres que le recuerdan sus orígenes emparentados con los lamas tibetanos y con los mongoles, en los que cree reconocer a Buda o a Cronos o a Saturno, a quien al fin identifica con Apollon. Es una pesadilla en que se mezclan la filosofía, el juicio por intentar matar al padre, lo que parece una experiencia extracorpórea $y$, finalmente, su transformación en bomba que explota. Alexander tratará de buscar explicaciones a la sensación de expansión experimentada por su amigo, a la que califica como un "estar fuera de sí": una pseudoalucinación, una sensación de abismo ("en términos modernistas"), una alegoría, la vibración de un cuerpo astral.

El autor también concede gran importancia a la descripción de las alucinaciones que se producen en el límite entre la vigilia y el sueño o por los efectos del alcohol, cuando los personajes están sometidos a un fuerte estrés. Alexander y Apollon atraviesan interesantes estados de delirio. La escalera del pobre edificio donde el primero tiene su buhardilla constituye una amenaza para él, observa extrañas sombras en la oscuridad, seres desconocidos que entran en su casa. En las mugrientas paredes de su cuarto ve aparecer el rostro de un mongol. En realidad teme su habitáculo por lo que puede encontrarse en él, pues este personaje, víctima de la pobreza y el alcohol, proyecta sus miedos en el espacio. En su peor delirio, al volver de la frustrada visita a Lippanchenko, tras escuchar un gran estruendo en las temidas escaleras, cree ver irrumpir en su propia casa nada menos que al Jinete de Bronce, que se derretirá en sus venas.

Los fantasmas de estos personajes están alimentados por sus conocimientos y sus obsesiones: filosofía, religión, antroposofía, historia de Rusia... El escritor ruso reflexiona irónicamente sobre el sutil y traspasable límite entre el mundo real y el mundo de las ideas. Como constata en un comentario de autor al final del primer capítulo, aunque los pensamientos no sean más que juegos cerebrales, la idea tiene una existencia propia. Esto explicaría los otros espacios de los personajes principales, cómo Nikolai, Alexander y Apollon conocen otra dimensión de la ciudad que se escapa del mundo empírico.

También ilustra la subjetividad espacial cómo la casa de los Ableukhov, escrutada por Anna, la madre, cuando entra por primera vez después de su regreso, es vista como un lugar donde nada ha cambiado, ni los viejos y tradicionales objetos decorativos, masculinos, fríos y sin vida, ni los sentimientos negativos que le inspiraba aquel lugar. Todo le recuerda que no fue feliz allí.

Pero el caso más notorio es la mirada geométrica del senador. El primer trayecto descrito en la novela es el realizado por Apollon desde su casa al Negociado. Desde su coche de caballos -cubo negro 
formado por cuatro tableros- observa la avenida Nevsky, cuya forma rectilínea atempera sus nervios; después de la línea, es el cuadrado lo que le da más tranquilidad. Los edificios se reducen a polígonos y paralelepípedos. No podemos encontrar una presentación mejor de una mente ordenada y sistemática.

\section{Conclusión}

Siguiendo a W. Benjamin, podemos decir que la ciudad, desde el siglo XIX, ha cambiado a los individuos; estos no son campesinos 0 terratenientes recién llegados, sino que la vida urbana implica una transformación antropológica. La ciudad incide sobre la vida de los seres que la habitan de forma que estos no son producto de una adaptación al medio sino, por el contrario, es el medio quien crea nuevos tipos. Entre los nuevos temas de la literatura simbolista rusa está la vida en la ciudad. Como señala Maliavina, "Sologúb, Blok y Biely reflejan la soledad y la perdición humana en el caos apocalíptico de la ciudad moderna, que convierte a las personas en seres aturdidos y estupefactos, transformando sus sentidos y pareceres" (2002: 135). En Petersburg vemos cómo los ciudadanos son ante todo individuos anónimos que son engullidos por la masa, organismo vivo y terrible. Son sombras que se materializan gracias al papel teúrgico del autor. Cuando se convierten en nombres propios toman la forma de seres en conflicto consigo mismos, antihéroes desorientados. Es el caso de los caracteres más importantes: tanto Nikolai como Alexander son dos intelectuales excesivamente reflexivos que tienden a aislarse, entregándose al estudio o a sus divagaciones. Apollon no consigue pensar con claridad, a pesar de su tendencia a la racionalidad. La interiorización de estos personajes se enriquece con la representación de su psiquismo, neurosis o incluso psicosis magníficamente descritas, que además se abren a interpretaciones simbólicas que van más allá del mundo físico. Por otra parte, sus experiencias urbanas son individuales, ya que la bella ciudad que acoge y cuida a sus ciudadanos también los vuelve solitarios y errantes. Nikolai, cual Hamlet de principios del siglo XX, se comporta como un neurótico pusilánime y miedoso incapaz de hacer frente a su destino.

Publicada antes que otras novelas modernistas europeas $-\mathrm{La}$ metamorfosis (1915), Ulysses (1922), Mrs. Dalloway (1925), Berlin Alexanderplatz (1929)-, Petersburg se alza como una novela precursora dentro de la experimentación vanguardista en narrativa. La obra maestra del autor ruso está construida con los recursos que serán empleados por los creadores modernistas: compresión del espacio y del tiempo como forma de centrar el interés en los movimientos mentales de los personajes y reflejar mejor la problemática del individuo moderno; la selección (sinécdoque) y deformación esperpéntica en la representación de los caracteres; la 
fragmentación de la historia en los diferentes tiempos y espacios de los seres de ficción; la irrupción de lo absurdo y de lo desconocido.

Otro ejemplo de renovación formal estaría en la creación de una prosa poética o rítmica; de hecho, los críticos están de acuerdo en que el lenguaje empleado por Biely es uno de los aspectos más destacados de la novela. Todas las citas que hemos ido extrayendo del texto muestran una prosa en la que abundan las figuras retóricas: paralelismos, anáforas, enumeraciones, animalizaciones, metonimias, metáforas, prosopopeyas, símiles $y$, sobre todo, el empleo de símbolos. El rojo es el símbolo de la revolución, por ello es odiado por el senador; aparece en las calles; es el color del dominó que viste Nikolai. El gris con que se identifica al alto dignatario, su vida monótona y sin interés. La evidente relación del "portador de insignias" con el dios Apolo, no solo por el nombre sino también por su amor a la razón y al orden, encuentra su símbolo en el sol que inunda sus estancias y se refleja en todos los objetos, justamente en la escena en que, tras el baile, debe mostrar su autoridad ante su hijo (capítulo quinto, 6). A Nikolai se le relaciona con Dionisos, tanto por su actitud caótica y privada de razón como por su experiencia "expansiva". El emblema distintivo de los Ableukhov es un unicornio atravesando a un caballero; el propio Nikolai lo interpreta cuando visualiza la muerte de su padre bajo los efectos de la bomba. La matrícula del coche del Senador es 1905, el año en que transcurre la historia. El mal tiempo atmosférico del "noxious October" cuando se describe la agitación y el miedo de los petersburguenses en los días previos a la manifestación, etc. Con toda seguridad se nos escapan otros símbolos de significado más esotérico que únicamente un lector "iniciado" podría descifrar. Asimismo, la traducción de la lengua rusa vuelve opacos los juegos de palabras así como las eufonías de una prosa rítmica que buscan acercar el lenguaje verbal al lenguaje musical. La osadía vanguardista también se muestra en la ruptura de los párrafos y su disposición en forma de escalera, de igual forma que Biely poeta rompía los versos de sus poemas y los dividía en sucesivos peldaños.

La voluntad rupturista de Biely se manifiesta en todos los niveles: la belleza formal del lenguaje (su prosa rítmica), la disposición gráfica de la prosa, el tema elegido, los procedimientos narrativos (diálogos muy largos, monólogo interior, descripciones que se fijan en los objetos personales), la fragmentación del hilo narrativo, la configuración de los personajes, la personificación de la ciudad, el profundo significado simbólico, el sentido del humor. Incluso las frecuentes intervenciones del narrador-autor, pudiendo resultar muy próximas a un narrador tradicional omnisciente, adquieren pleno significado si, atendiendo a las palabras del autor, vemos cómo la creación literaria no deja de ser un "juego mental" del escritor gracias al cual el relato y sus entes de ficción cobran vida y, 
por tanto, existen, en cuyo caso, su grado de existencia, ¿por qué no está al mismo nivel que el nuestro?

\section{Bibliografía}

BIELY, Andrei (1987): St. Petersburg. Edited by John Courton. New York: Grove Press.

- (2002): Petersburgo. Madrid: Santillana.

- (2009): Petersburgo. Madrid: Akal.

DE CERTEAU, Michel (1994): "Walking on the city", en DURING, Simon (ed.), The Cultural Studies Reader. London: Routledge.

GENETTE, Gérard (1989): Figuras III. Barcelona: Lumen.

JOYCE, James (1998): Ulises. Edición de José María Valverde. Barcelona: Lumen.

LÓPEZ-VARELA, Asunción (2010): "Spatiotemporal topography and metaphorical border-crossings in English literature". Sun Yat-sen Journal of Humanities 28, junio de 2010, pp. 1-17.

LÓPEZ-VARELA, Asunción; y NET, Mariana (2009): Real and Virtual Cities: Intertextual and Intermedial Mindscapes. Bucharest: Univers Enciclopedics.

LÓPEZ-VARELA, Asunción; y TÖTÖSY DE ZEPETNEK, Steven (2009): "Real and Imagined Urban Spaces and Transformations in/of Western Society", en Mariana Net (ed.), Urban Spaces and Transformations in/of Western Society, TRANS. 16 Internet-Zeitschrift für Kulturwissenschaften / Internet Journal of Cultural Studies / Journal of the Research Institute for Austrian and International Literature and Cultural Studies (INST).

MALIAVINA, Svetlana (1997): "Simbolismo ruso", en Historia de las literaturas eslavas, pp. 1195-1217. Madrid: Cátedra.

- (2002): "El simbolismo ruso. El origen y la originalidad del movimiento". Eslavística Complutense, vol. 2, pp. 127-149.

PRESA GONZÁLEZ, Fernando (coord.) (1997): Historia de las literaturas eslavas. Madrid: Cátedra.

RENDUELOS, César; y USEROS, Ana (2010): Atlas (CD-Rom). Constelaciones (DVD). Walter Benjamin (estuche). Madrid: Círculo de Bellas Artes.

ZHANG, Yingjin (1996): The City in Modern Chinese Literature and Film. Standford: Standford University Press. 